\title{
Risk and Timing of Neutropenic Events in Adult Cancer Patients Receiving Chemotherapy: The Results of a Prospective Nationwide Study of Oncology Practice
}

\author{
Jeffrey Crawford, MD; ${ }^{a}$ David C. Dale, MD; ${ }^{b}$ Nicole M. Kuderer, MD; Eva Culakova, PhD; ${ }^{a}$ \\ Marek S. Poniewierski, MD; ${ }^{a}$ Debra Wolff, MS, PCNP; and Gary H. Lyman, MD, MPH, ${ }^{a}$ \\ Durham, North Carolina, and Seattle, Washington
}

\section{Key Words}

Neutropenia, fever, infection, chemotherapy, cancer

\begin{abstract}
This study was undertaken to describe the relationship between the occurrence and timing of neutropenic events and chemotherapy treatment in a community-based population of patients with cancer. The study included 2962 patients with breast, lung, colorectal, lymphoma, and ovarian cancers from a prospective U.S. registry of patients initiating a new chemotherapy regimen. Detailed patient-, disease-, and treatment-related data, including toxicities, were captured at baseline, the beginning of each cycle, and each midcycle blood draw for up to 4 cycles of treatment. Primary outcomes included febrile neutropenia (FN), severe neutropenia without fever/ infection, and relative dose intensity (RDI). Thirty-seven percent of patients were aged 65 years or older, $43.5 \%$ had an Eastern Cooperative Oncology Group performance status of 1 or greater, and $27 \%$ had 1 or more comorbidities. Reductions in RDI to less than $85 \%$ of standard in the first cycle were planned in $23.6 \%$ of patients, whereas primary colony-stimulating factor prophylaxis was used in $18.2 \%$. In the first 3 cycles of treatment, $10.7 \%$ of patients experienced $\mathrm{FN}$, with most of these events $(58.9 \%)$ occurring in the first cycle. This first-cycle pattern was consistently observed despite wide variations in event rates by tumor type, disease stage, chemotherapy regimen and dose, and patient characteristics. Despite
\end{abstract}

From 'Duke University, Durham, North Carolina, and 'University of Washington, Seattle, Washington.

Submitted May 23, 2007; accepted for publication August 6, 2007. This article was supported in part by a research grant from Amgen Inc., Thousand Oaks, California.

Presented at the American Society of Hematology $46^{\text {th }}$ Annual

Meeting, December 4-7, 2004, San Diego, California.

Correspondence: Gary H Lyman, MD, MPH, Duke University

Medical Center, DUMC Box 3645, Durham, NC 27710.

E-mail: Gary.Lyman@Duke.edu frequent planned reductions from standard RDI, the incidence of FN remains high in community oncology practice in the United States. Improved methods of pretreatment assessment of patient risk factors for neutropenia are needed. (JNCCN 2008;6:109-118)

In current clinical practice, the use of chemotherapy is associated with a high risk for neutropenia and associated complications. Febrile neutropenia (FN), the most serious manifestation of neutropenia, is associated with early mortality, lengthy hospitalizations, and high medical costs. ${ }^{1,2}$ In addition, both the risk for and actual occurrence of FN drive chemotherapy dose reductions and delays that can potentially compromise the goals of cancer treatment..$^{3-6}$ The resulting reduced dose intensity lowers the risk for neutropenia and other toxicities but may also worsen outcomes for patients with chemotherapyresponsive malignancies. ${ }^{5-15}$

Despite its importance, chemotherapy-induced neutropenia has not been well studied in community-based populations. Age, comorbidities, performance status, and disease stage as risk factors for neutropenic complications are supported by retrospective evidence. ${ }^{16}$ Studies in nonHodgkin's lymphoma (NHL) ${ }^{17-19}$ and breast cancer ${ }^{20}$ have shown that the risk for neutropenia and its complications, including $\mathrm{FN}$, is greatest during the first cycle. To further improve understanding of the risk and timing of neutropenic events, the authors implemented a large community-based registry of patients initiating chemotherapy for commonly encountered cancers. This prospective study confirms that a predominantly first-cycle pattern of 
Crawford et al.

neutropenic events applies generally to both lymphomas and common solid tumors.

\section{Patients and Methods}

\section{Registry Design and Patient Selection}

This registry study, undertaken by the Awareness of Neutropenia in Chemotherapy Study Group, was designed to prospectively collect data on typical patients undergoing chemotherapy in the community oncology setting. Using a nationwide inventory of 2382 oncology practices, sites were grouped into 5 quintiles based on volume statistics and 4 geographic regions (Northeast, Southern, Central, and Western United States). Stratified random sampling based on size of practice and geographic location was used to select participating sites. All sites were approved by the Institutional Review Board (IRB) and all patients signed an IRB-approved informed consent. To avoid selection bias, sites were instructed to enroll consecutive patients. Site management and data collection were coordinated by an independent clinical research organization, and neither the investigators nor the funding agency had direct knowledge of the participating sites. The data reside at the Study Coordinating Center where all data analyses, reporting, and interpretation were performed independently of the funding agency. The investigators retained all rights to data analysis and interpretation, decision to publish, and final contents of this publication.

Specific treatment interventions or alterations in therapy were not mandated, required, or implied by the study, except for a midcycle blood draw in each cycle to obtain blood counts and differentials at the expected time of nadir, which was clinician-determined. Any adult patient starting a new chemotherapy regimen for any solid tumor, lymphoma, or chronic leukemia was eligible. Life expectancy of at least 3 months was required, with at least 4 cycles of chemotherapy planned at the outset. Age was restricted to patients 18 years or older, with no upper limit. Prior chemotherapy and concurrent radiation therapy were permitted. Exclusion criteria were limited and included myeloma, HIV infection, or a history of stem cell (bone marrow, peripheral blood, or cord blood) transplantation. Patients undergoing concurrent myelosuppressive cytotoxic, biologic, or immunologic therapy for other conditions (e.g., methotrexate for arthritis) and those participating in blinded trials of known myelosuppressive drugs were also ineligible. The primary tumor types representing the a priori focus of this study and the analysis presented here include breast, lung (non-small cell [NSCLC], small cell [SCLC]), colorectal, lymphoma (NHL and Hodgkin disease [HD]), and ovarian cancers.

\section{Study Variables, Operational Definitions, and Study Outcomes}

Pretreatment and cycle-specific data were collected for each patient on up to 4 consecutive cycles of chemotherapy at baseline, the beginning of each cycle of chemotherapy, and each midcycle blood draw. Baseline demographics and clinical variables included age, gender, ethnicity, employment and educational status, insurance, zip code, ECOG performance status (PS), body surface area (BSA), cancer stage using the American Joint Committee on Cancer criteria, past cancer history, prior treatment, selected concomitant medications, and planned chemotherapy treatment. Cycle and midcycle data were collected on hematology and chemistry laboratory tests, drugs and dosing information, adverse events, and other treatment information. Standard dose and schedule for each regimen were established based on relevant published clinical trial reports. The dose intensity was estimated based on the BSA reported and used for each patient. Relative dose intensity (RDI) could be calculated for more than $90 \%$ of patients by taking a ratio of either planned or actual doses per unit time to the standard for each agent within a regimen. RDI was divided into planned reductions, prescribed at the initiation of treatment, and unplanned reductions, which were subsequent changes in dose and schedule occurring during the course of therapy. The average planned, unplanned, and total dose reductions, and treatment delays and RDI were then calculated across all myelosuppressive agents in each regimen.

The primary outcomes of interest for this analysis were FN (fever/infection and absolute neutrophil count $[\mathrm{ANC}]<1000 / \mathrm{mm}^{3}$ ), severe neutropenia (SN) (ANC $<500 / \mathrm{mm}^{3}$ without fever/infection), and planned RDI of less than $85 \%$. The results are shown for $\mathrm{FN}$ alone and for the composite end point of FN or SN. Because information on febrile episodes was captured for the previous cycle in the case report forms for the following cycle, and because these forms were completed for up to 4 cycles, FN events in the fourth cycle were not fully captured. Therefore, the results are reported for the first 3 cycles only. Data were available for all 3 cycles in more than $80 \%$ of patients, with only $2 \%$ lost to follow-up. As a secondary outcome, the 
Risk and Timing of Neutropenic Events

\begin{tabular}{|c|c|c|c|c|c|c|c|}
\hline \multirow[b]{2}{*}{ Characteristic } & \multicolumn{3}{|c|}{ All Patients } & \multicolumn{2}{|c|}{ Stages I-III* } & \multicolumn{2}{|r|}{ Stage IV } \\
\hline & n (\%) & $\begin{array}{l}\text { FN or SN } \\
\text { in Cycle } 1\end{array}$ & $\begin{array}{l}\text { Planned } \\
\text { RDI } \geq 85 \% \\
(\%)\end{array}$ & $\mathbf{n}$ & $\begin{array}{l}\text { Planned } \\
\text { RDI } \geq 85 \% \\
\text { (\%) }\end{array}$ & $\mathrm{n}$ & $\begin{array}{l}\text { Planned } \\
\text { RDI } \geq 85 \% \\
(\%)\end{array}$ \\
\hline $\begin{array}{l}\text { All patients } \\
\text { Age }(y)\end{array}$ & $2692(100 \%)$ & 20.1 & 76.4 & 1824 & 80.2 & 868 & 68.4 \\
\hline $18-64$ & 1692 (62.9) & 22.2 & 81.0 & 1271 & 83.9 & 421 & 72.2 \\
\hline $65-74$ & $649(24.1)$ & 16.9 & 70.3 & 363 & 75.2 & 286 & 64.0 \\
\hline$\geq 75$ & $351(13.0)$ & 16.2 & 65.2 & 190 & 64.2 & 161 & 66.5 \\
\hline \multicolumn{8}{|l|}{ Gender } \\
\hline Female & $1924(71.5)$ & 24.0 & 78.2 & 1446 & 82.4 & 478 & 65.7 \\
\hline Male & $768(28.5)$ & 10.4 & 71.7 & 378 & 71.7 & 390 & 71.8 \\
\hline \multicolumn{8}{|l|}{ Race } \\
\hline White & $2265(84.1)$ & 20.0 & 76.2 & 1532 & 80.3 & 733 & 67.5 \\
\hline Black & $279(10.4)$ & 20.1 & 74.9 & 180 & 76.1 & 99 & 72.7 \\
\hline Other & $148(5.5)$ & 22.3 & 82.4 & 112 & 84.8 & 36 & 75.0 \\
\hline \multicolumn{8}{|l|}{ Body surface area } \\
\hline$\leq 2 \mathrm{~m}^{2}$ & 2182 (81.1) & 21.6 & 77.5 & 1505 & 82.2 & 677 & 66.9 \\
\hline$>2 \mathrm{~m}^{2}$ & $510(18.9)$ & 13.9 & 71.8 & 319 & 70.5 & 191 & 73.8 \\
\hline \multicolumn{8}{|l|}{ Cancer type } \\
\hline Breast & $1138(42.3)$ & 30.7 & 85.0 & 970 & 88.2 & 168 & 66.1 \\
\hline Non-small cell lung & $484(18.0)$ & 7.2 & 64.3 & 218 & 58.7 & 266 & 68.8 \\
\hline Small cell lung & $156(5.8)$ & 29.5 & 68.6 & 66 & 68.2 & 90 & 68.9 \\
\hline Colorectal cancer & $379(14.1)$ & 5.3 & 75.7 & 192 & 80.2 & 187 & 71.1 \\
\hline Non-Hodgkin's lymphoma & $257(9.5)$ & 21.8 & 78.2 & 165 & 77.0 & 92 & 80.4 \\
\hline Hodgkin disease & $55(2.0)$ & 23.6 & 96.4 & 52 & 100.0 & 3 & 33.3 \\
\hline Ovarian & $223(8.3)$ & 10.3 & 58.3 & 161 & 62.1 & 62 & 48.4 \\
\hline \multicolumn{8}{|l|}{ Disease stage } \\
\hline 1 & $359(13.3)$ & 29.5 & 81.3 & 359 & 81.3 & - & - \\
\hline II & $755(28.0)$ & 26.9 & 85.0 & 755 & 85.0 & - & - \\
\hline III & $685(25.4)$ & 15.5 & 74.6 & 685 & 74.6 & - & - \\
\hline IV & $868(32.2)$ & 13.6 & 68.4 & - & - & 868 & 68.4 \\
\hline Unknown & $25(0.9)$ & 36.0 & 68.0 & 25 & 68.0 & & \\
\hline Prior chemotherapy & $600(22.3)$ & 13.5 & 65.8 & 246 & 69.9 & 354 & 63.0 \\
\hline Prior radiotherapy & $364(13.5)$ & 15.7 & 68.4 & 154 & 72.1 & 210 & 65.7 \\
\hline \multicolumn{7}{|l|}{ ECOG performance status } & 74.1 \\
\hline 0 & $1521(56.5)$ & 22.2 & 78.6 & 1210 & 81.7 & 311 & 66.9 \\
\hline 1 & $959(35.6)$ & 17.8 & 75.0 & 542 & 78.0 & 417 & 71.0 \\
\hline$\geq 2$ & $212(7.9)$ & 15.6 & 66.5 & 72 & 70.8 & 140 & 64.3 \\
\hline \multicolumn{8}{|l|}{ Comorbidities } \\
\hline Diabetes mellitus & $296(11.0)$ & 18.9 & 71.3 & 182 & 78.0 & 114 & 60.5 \\
\hline Chronic lung disease & $230(8.5)$ & 18.7 & 68.3 & 119 & 68.1 & 111 & 68.5 \\
\hline
\end{tabular}


Crawford et al.

\begin{tabular}{|c|c|c|c|c|c|c|c|}
\hline \multirow[b]{2}{*}{ Characteristic } & \multicolumn{3}{|c|}{ All Patients } & \multicolumn{2}{|c|}{ Stages I-III* } & \multicolumn{2}{|c|}{ Stage IV } \\
\hline & n (\%) & $\begin{array}{l}\text { FN or SN } \\
\text { in Cycle } 1\end{array}$ & $\begin{array}{l}\text { Planned } \\
\text { RDI } \geq 85 \% \\
\text { (\%) }\end{array}$ & $\mathbf{n}$ & $\begin{array}{l}\text { Planned } \\
\text { RDI } \geq 85 \% \\
\text { (\%) }\end{array}$ & $\mathbf{n}$ & $\begin{array}{l}\text { Planned } \\
\text { RDI } \geq 85 \% \\
(\%)\end{array}$ \\
\hline Cardiovascular disease & $199(7.4)$ & 14.1 & 68.8 & 110 & 71.8 & 89 & 65.2 \\
\hline Ulcer disease & $85(3.2)$ & 10.6 & 67.1 & 58 & 75.9 & 27 & 48.1 \\
\hline \multicolumn{8}{|l|}{ No. of comorbidities ${ }^{\dagger}$} \\
\hline 0 & $1966(73.0)$ & 21.0 & 78.7 & 1401 & 82.0 & 565 & 70.6 \\
\hline 1 & $548(20.4)$ & 19.5 & 71.2 & 323 & 75.9 & 225 & 64.4 \\
\hline$\geq 2$ & $178(6.6)$ & 12.9 & 66.3 & 100 & 68.0 & 78 & 64.1 \\
\hline \multicolumn{8}{|l|}{ Chemotherapeutic } \\
\hline Anthracyclines & 1065 (39.6) & 36.0 & 87.5 & 961 & 88.6 & 104 & 77.9 \\
\hline Platinums & $827(30.7)$ & 11.4 & 65.7 & 411 & 62.8 & 416 & 68.5 \\
\hline Taxanes & $810(30.1)$ & 13.7 & 68.6 & 477 & 70.2 & 333 & 66.4 \\
\hline \multicolumn{8}{|l|}{ No. of agents } \\
\hline 1 & $516(19.2)$ & 7.4 & 70.5 & 282 & 76.2 & 234 & 63.7 \\
\hline 2 & $1758(65.3)$ & 21.0 & 74.9 & 1163 & 77.8 & 595 & 69.2 \\
\hline 3 & $358(13.3)$ & 33.2 & 88.5 & 325 & 88.6 & 33 & 87.9 \\
\hline$\geq 4$ & $60(2.2)$ & 25.0 & 96.7 & 54 & 100.0 & 6 & 66.7 \\
\hline Primary CSF prophylaxis & $490(18.2)$ & 8.0 & 82.9 & 359 & 86.4 & 131 & 73.3 \\
\hline
\end{tabular}

*Unknown stage $(n=25)$ was grouped with stage I-III.

${ }^{\dagger}$ Comorbidities captured include congestive heart failure, myocardial infarction, peripheral vascular disease, hemiplegia/paraplegia, cerebrovascular disease, diabetes, renal disease, ulcer, liver disease, and connective tissue disease.

Abbreviations: CSF, colony-stimulating factor; ECOG, European Cooperative Oncology Group; FN, febrile neutropenia; RDI,

relative dose intensity; $\mathrm{SN}$, severe neutropenia.

use of colony-stimulating factor (CSF) was defined as either primary or secondary prophylaxis. Primary CSF prophylaxis was defined as CSF use planned at the beginning of the first cycle or before a neutropenic event within the first cycle, whereas secondary CSF prophylaxis was defined as CSF prescribed in response to a neutropenic event.

Data quality control efforts included edit and logic checks, weekly queries and missing page reports to each site, countercheck questions incorporated into the case report form design, double data entry, site monitoring visits, and further quality checks on interim data transfers.

\section{Statistical Methods}

After extensive quality checks for data integrity, variable distributions and summary measures were generated, including mean, median, range, and standard deviation range for continuous variables and frequencies for categorical variables. Selected continuous variables were dichotomized based on clinically relevant cut points (e.g., RDI $<85 \%$, BSA $>2 \mathrm{~m}^{2}$ ). The chi-square test was used to assess the association between categorical variables. A $P$ value of less than .05 was used to denote statistical significance. All data analysis was performed solely by the investigators at the Coordinating Center at Duke University.

\section{Results}

\section{Patient Population}

Between March 2002 and March 2005, a total of 3593 patients initiating a new chemotherapy regimen were prospectively enrolled at 115 practice sites throughout the United States. Table 1 shows the patient, disease, and treatment characteristics for the 2692 patients with 1 of the 5 major cancer types included in this analysis. Mean and median ages were 59.4 and 60.0 years, respectively, with more than one third of patients aged 65 years or older $(37.1 \%)$. The most 
Risk and Timing of Neutropenic Events

common comorbidities were diabetes (11\%), chronic lung disease $(8.5 \%)$, and cardiovascular disease (7.4\%). More than half (53.6\%) of patients had stage II or III disease, approximately a third (32.2\%) had stage IV disease, and more than $40 \%$ had an ECOG PS of 1 or more. An anthracycline was the most commonly used myelosuppressive agent $(39.6 \%)$, with a platinum $(30.7 \%)$ or taxane $(30.1 \%)$ also frequently used. Most patients $(80.8 \%)$ received a regimen containing 2 or more myelosuppressive agents. Although patients with active infection were specifically excluded from study entry, subsequent infections and antibiotic use was captured. Over the first 3 cycles of treatment, infection was reported in 439 patients (16\%), including 296 (11\%) with bacterial infections, 69 (2.5\%) with fungal infection, and the remaining represented by viral or unknown infections. Detailed microbiologic culture results were not available. Antibiotic use during therapy was reported in 751 patients $(27.9 \%)$ including $121(4.5 \%)$ at or before the initiation of chemotherapy. The available data do not permit discrimination between the use of antibiotics for treatment versus prophylaxis for subsequent cycles.

\section{Timing of Neutropenic Events}

FN events were documented in 287 patients (10.7\%), whereas 788 (29.3\%) experienced either FN or SN during the first 3 cycles of chemotherapy. For both outcomes, the highest rate of initial events occurred in the first cycle (Figure 1), with the number of initial events decreasing in subsequent cycles. Of patients experiencing FN, 58.9\% had their initial event in cycle 1. Similarly, of patients experiencing either $\mathrm{FN}$ or $\mathrm{SN}, 68.8 \%$ had their initial occurrence in the first cycle. This first cycle trend was found across tumor types, with first-cycle events ranging from $50.0 \%$ of all such events in NSCLC to $71.4 \%$ in SCLC and $80.0 \%$ in colorectal cancer (Figure 2). SN events followed a similar, predominantly first-cycle pattern (see Figure 2). The overall incidence of neutropenic events varied between tumor types, with FN rates over 3 cycles of more than 10\% in SCLC (17.9\%), breast cancer (15.2\%), NHL (14.0\%), and HD (12.7\%), and FN/SN rates over 3 cycles of more than $20 \%$ in breast cancer $(41.7 \%)$, SCLC $(39.7 \%)$, NHL (35.4\%), HD (30.9\%), and ovarian cancer (22.4\%).

This first-cycle pattern was observed regardless of the planned RDI (Figure 3). RDI, however, influenced the incidence of neutropenic events observed. With the exception of SCLC, planned RDI of less
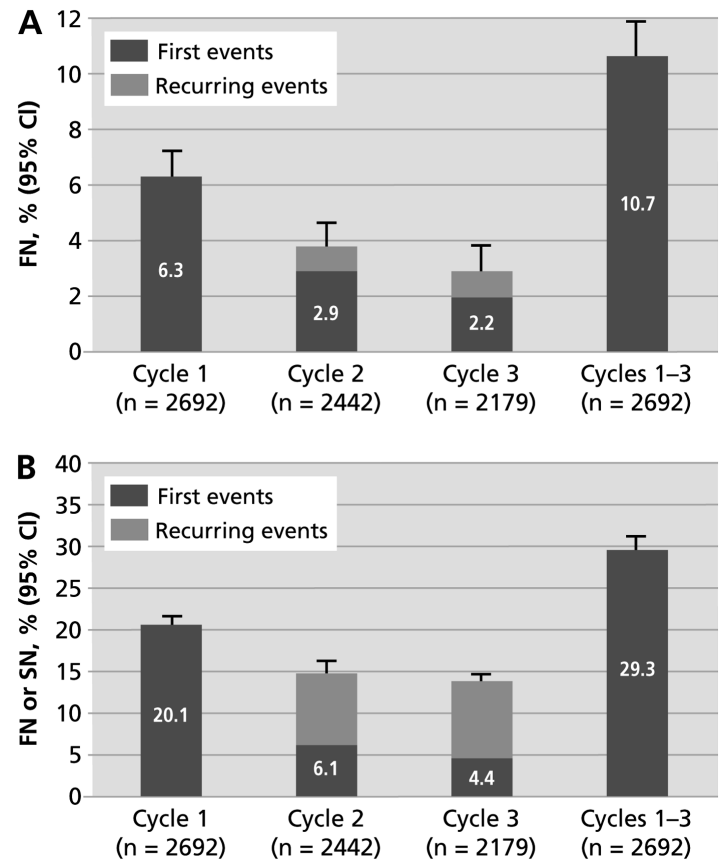

Figure 1 (A) Incidence of first and recurring FN (fever/infection and ANC $<1000 / \mathrm{mm}^{3}$ ). (B) FN or SN (ANC $<500 / \mathrm{mm}^{3}$ without fever/ infection) by cycle.

Abbreviations: ANC, absolute neutrophil count; CI, confidence interval; FN, febrile neutropenia; SN, severe neutropenia.

than $85 \%$ was associated with approximately half the events observed with planned RDI of $85 \%$ or more (see Figure 3). Besides RDI, other factors that seemed to influence the incidence of neutropenic events included disease stage and treatment with primary CSF prophylaxis. Disease stage had a variable association depending on cancer type, with stage IV disease associated with lower rates of events in some cancer types

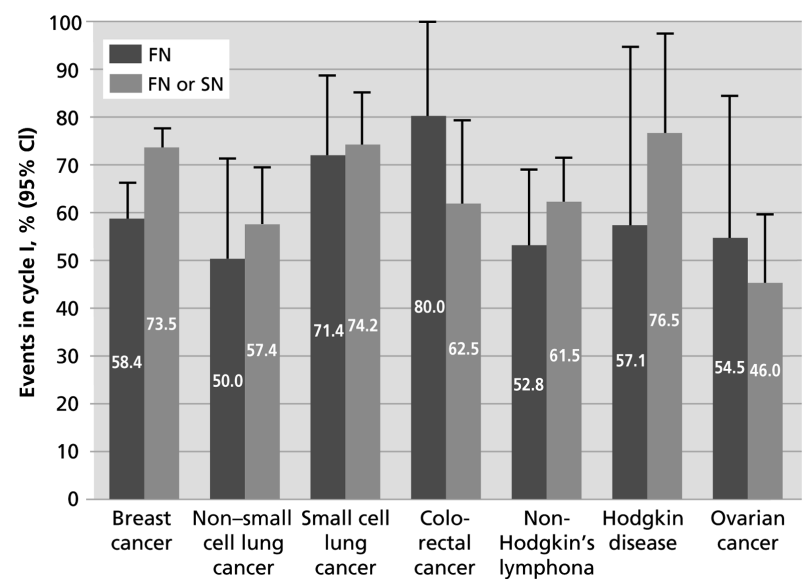

Figure 2 Proportion of first FN (fever/infection and ANC $<1000 / \mathrm{mm}^{3}$ ) and $\mathrm{FN}$ or SN (ANC $<500 / \mathrm{mm}^{3}$ without fever/infection) events in cycle 1 , by cancer type.

Abbreviations: ANC, absolute neutrophil count; CI, confidence interval; FN, febrile neutropenia; $\mathrm{SN}$, severe neutropenia. 
Crawford et al.
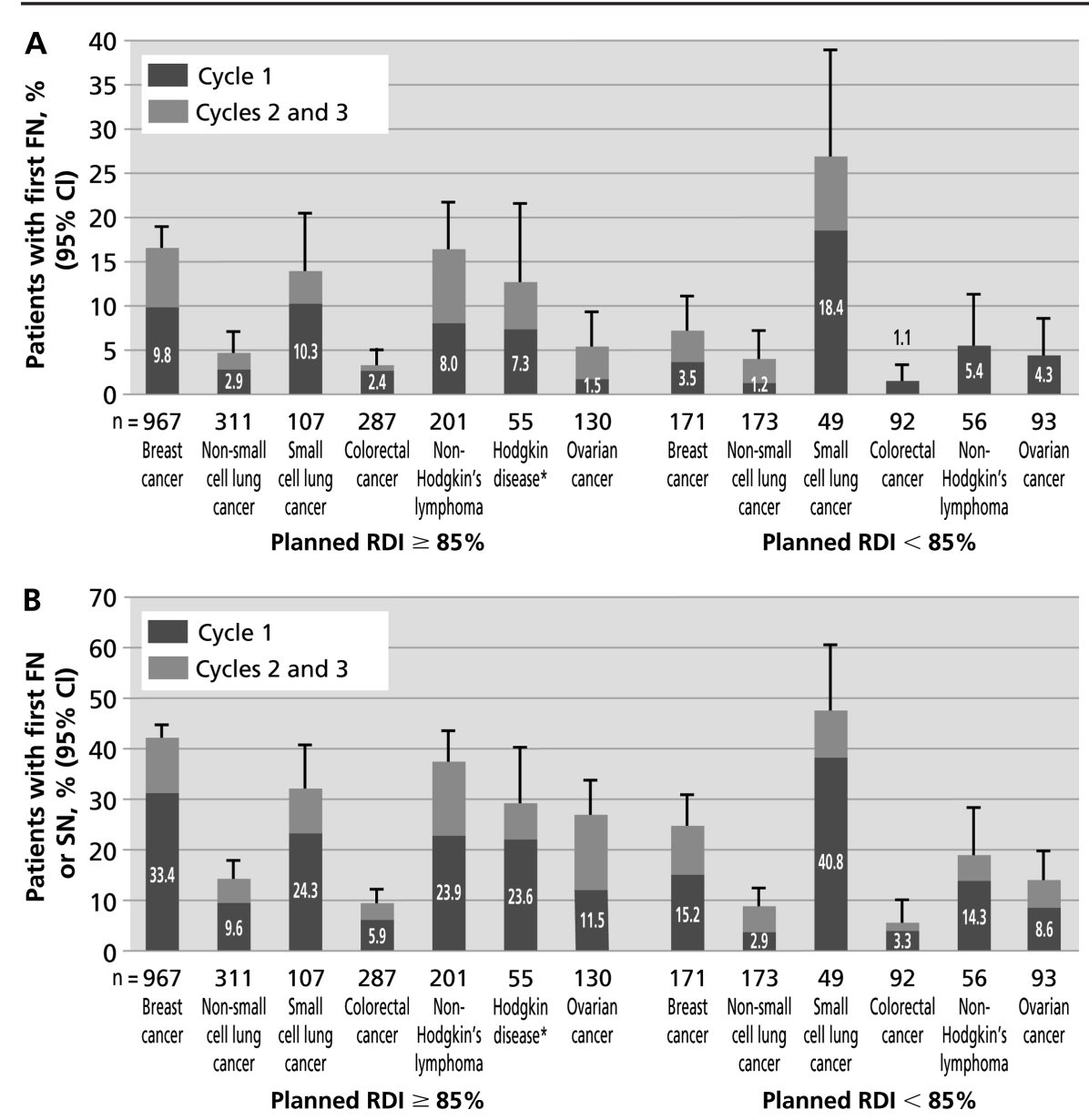

Figure 3 (A) Incidence of first FN (fever/infection and ANC $<1000 / \mathrm{mm}^{3}$ ), and (B) FN or SN (ANC $<500 / \mathrm{mm}^{3}$ without fever/infection) by cancer type, cycle, and planned RDI $<85 \%$ and $\geq 85 \%$ (based on reference standards).

*Only 2 patients with Hodgkin disease had planned RDI < 85\%.

Abbreviations: ANC, absolute neutrophil count; CI, confidence interval; FN, febrile neutropenia; RDI, relative dose intensity; $\mathrm{SN}$, severe neutropenia.

(colorectal, breast, and NHL) but higher event rates in others (NSCLC, SCLC; see Table 2). The use of primary CSF prophylaxis was associated with lower rates of neutropenic events across cancer types (see Table 2 ). FN rates were highest in patients with planned RDI of $85 \%$ or more and no primary CSF prophylaxis, reaching $19.4 \%$ and $23.7 \%$ in patients with early-stage breast cancer and NHL, respectively.

\section{RDI}

The demographic information of the patient population and proportion of patients with planned RDI of $85 \%$ or more of the reference standard are shown in Table 1. Overall, almost a quarter of patients (23.6\%) were prescribed a reduced RDI ( $<85 \%$ of standard). The likelihood of receiving a reduced RDI varied with cancer type, with planned RDI of less than $85 \%$ most common in ovarian cancer $(41.7 \%)$, followed by
NSCLC (35.7\%), SCLC $(31.4 \%)$, colorectal cancer (24.3\%), NHL (21.8\%), breast cancer $(15.0 \%)$, and HD (3.6\%). Besides tumor type, additional factors associated with an increased likelihood of RDI of less than $85 \%$ included increasing age, BSA greater than $2 \mathrm{~m}^{2}$, stage IV disease, prior therapy, ECOG PS of 2 or more, and comorbidities. Regarding chemotherapy type, anthracycline-containing regimens were more commonly delivered at RDI of $85 \%$ or more, whereas platinum- and taxanecontaining regimens were less frequently prescribed at RDI of $85 \%$ or more. RDI less than $85 \%$ was more common with monotherapy, whereas regimens of 3 and 4 myelosuppressive drugs were more likely to be delivered at RDI of $85 \%$ or more. The use of primary CSF prophylaxis was more common with planned RDI of $85 \%$ or more, and in patients with stage I through III disease compared with stage IV.

Of the 2692 patients, 513 (19\%) did not reach cycle 4 (Table 3 ). Reasons for early chemotherapy termination included early death in 49 (9.5\%), progressive disease in 95 (18.5\%), treatment-related toxicity in 133 (25.9\%), protocol violation in 99 (19.3\%), lost to follow-up in $57(11.1 \%)$, and other causes in $80(15.6 \%)$.

\section{First-Cycle Neutropenic Events and Subsequent Treatment}

First-cycle FN was significantly associated with chemotherapy modifications in subsequent cycles. Patients with first-cycle FN had twice as many unplanned dose reductions in cycle 2 than patients with first-cycle SN or without FN or SN (11.1\% vs. 5.9\% and $5.4 \% ; P=.0033$; Figure 4$)$. Unplanned delays in the initiation of cycle 2 were also significantly increased in patients with first-cycle FN $(22.2 \%$ vs. $13.5 \%$ and $13.0 \% ; P=.0010)$. These changes resulted 
Risk and Timing of Neutropenic Events

Table 2 Neutropenic Events in Cycles 1-3

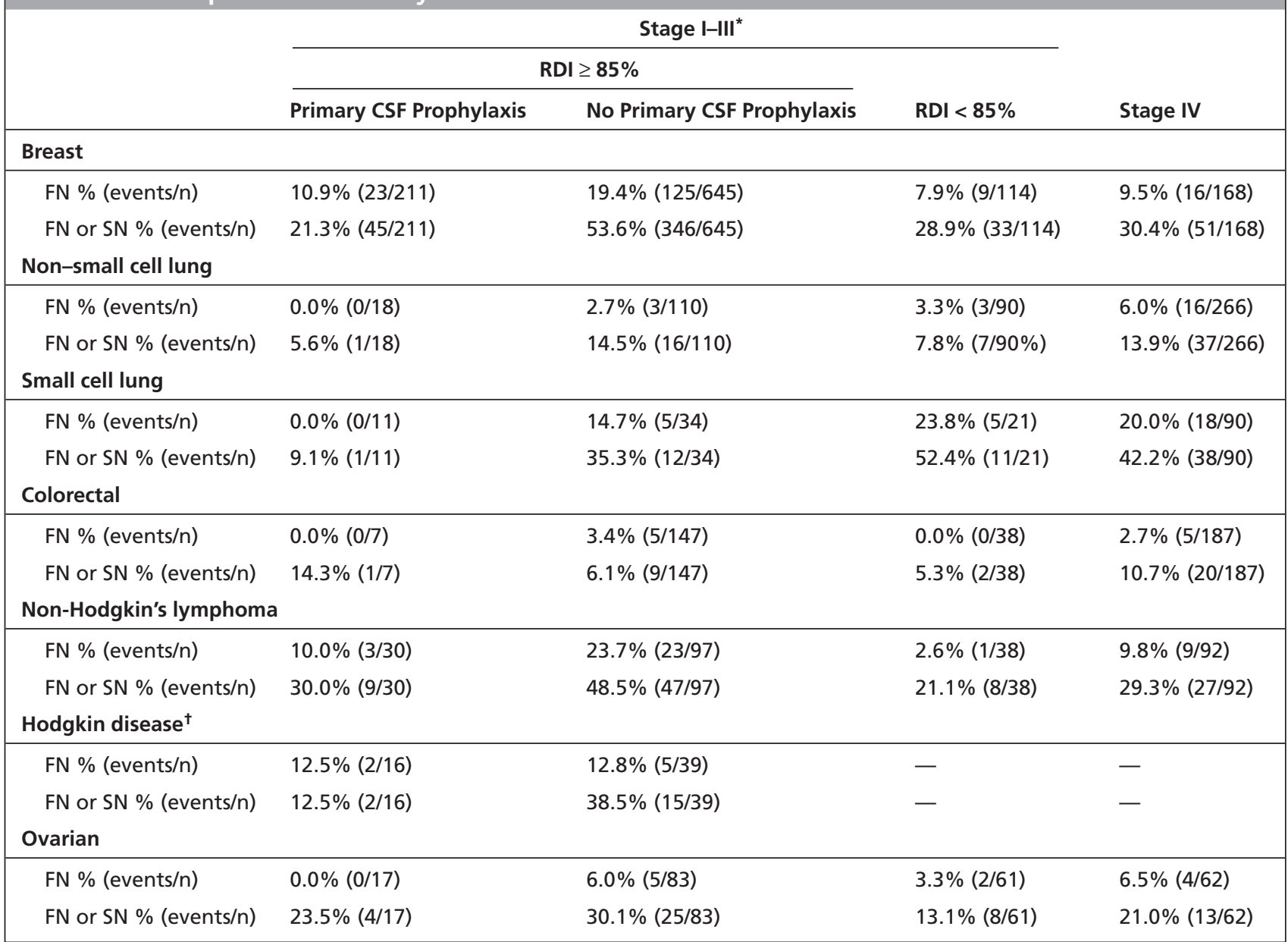

*Unknown stage $(n=25)$ was grouped with stage I-III.

${ }^{\dagger}$ All but 3 patients with Hodgkin disease had early-stage disease and only 2 had planned RDI < 85\%.

Abbreviations: CSF, colony-stimulating factor; FN, febrile neutropenia; RDI, relative dose intensity; SN, severe neutropenia.

in an unplanned RDI of less than $85 \%$ in $38.3 \%$ of patients with first-cycle FN compared with $23.8 \%$ of patients with first-cycle $\mathrm{SN}$ and $25.0 \%$ without $\mathrm{FN}$ or $\mathrm{SN}(P=.0002)$. In contrast, patients with first-cycle $\mathrm{SN}$ without fever did not experience differences in chemotherapy modifications compared with patients without either FN or SN.

First-cycle FN was also a significant driver of secondary CSF prophylaxis, with almost 3 times as many patients receiving CSF prophylaxis in cycle $2 \mathrm{com}$ pared with those without first-cycle FN or SN $(77.8 \%$ vs. $28.8 \% ; P<.0001)$. Furthermore, twice the number of patients with first-cycle SN without fever received CSF prophylaxis in cycle 2 compared with those without first-cycle FN or SN ( $56.5 \%$ vs. $28.8 \%$; $P<.0001)$. Overall, prophylactic CSF use increased with each cycle from less than $20 \%$ of patients in the first cycle to more than $40 \%$ by the third cycle. In contrast, unplanned dose reductions and delays generally increased only modestly with subsequent cycles.

\section{Discussion}

This registry study represents the first large-scale prospective study of the delivery of chemotherapy across the spectrum of oncology practices in the United States. Unlike clinical trials restricted to narrowly defined patient populations, this registry was intended to be as inclusive as possible to capture the impact of cancer chemotherapy on neutropenia and related complications in community oncology practice. As a result, $37 \%$ of these patients were aged 65 years or older, $43.5 \%$ had an ECOG PS of 1 or more, and $27 \%$ had 1 or more comorbidities. More than 
Crawford et al.

\begin{tabular}{|c|c|c|c|}
\hline & & $\begin{array}{l}\text { FN or SN in } \\
\text { Cycle } 1(\%)\end{array}$ & $\begin{array}{l}\text { FN or SN in } \\
\text { Cycles 1-3 (\%) }\end{array}$ \\
\hline Reached nadir of cycle $3(n=2179)$ & & 21.3 & 31.8 \\
\hline Did not reach nadir of cycle $3(n=513)$ & & 15.2 & 18.5 \\
\hline \multicolumn{4}{|l|}{ Reason for drop-out } \\
\hline \multirow[t]{2}{*}{ Death } & Progressive disease $(n=35)$ & 8.6 & 8.6 \\
\hline & Other complication $(n=14)$ & 21.1 & 31.6 \\
\hline \multirow[t]{4}{*}{ Medical reason } & Progressive disease $(n=95)$ & 2.1 & 6.4 \\
\hline & Toxicity $(n=82)$ & 22.8 & 27.8 \\
\hline & Patient-requested withdrawal $(n=51)$ & 21.6 & 21.6 \\
\hline & Other $(n=80)$ & 17.5 & 21.3 \\
\hline Protocol-related (administrative) $(\mathrm{n}=99)$ & & 20.6 & 23.7 \\
\hline Lost to follow-up/unknown $(n=57)$ & & 10.3 & 12.1 \\
\hline
\end{tabular}

Abbreviations: FN, febrile neutropenia; SN, severe neutropenia.

$80 \%$ of patients were treated with multiagent chemotherapy and $22 \%$ had undergone a prior course of chemotherapy.

Despite a planned reduction in RDI of less than $85 \%$ for nearly a quarter of patients, the overall rate of FN over the first 3 cycles of treatment was $10.7 \%$, varying greatly by cancer type from $2.6 \%$ in colorectal cancer to $17.9 \%$ in SCLC. In the tumor types studied, most initial episodes occurred during the first cycle of chem-otherapy, averaging $58.9 \%$ and $68.8 \%$ of $\mathrm{FN}$ and either FN or SN events, respectively. This first-cycle pattern was consistently observed regardless of disease stage and chemotherapy regimen and dose.

The results showing a substantial proportion of patients experiencing a planned reduction in RDI less than $85 \%$ starting with the first cycle are consistent with earlier observations. ${ }^{5,6}$ For some tumor types, including SCLC (31.4\%), NSCLC (35.7\%), and ovarian cancer $(41.7 \%)$, this proportion was very high. In settings where most patients were treated with curative intent, this proportion was lower (HD, 3.6\%; breast cancer, $15.0 \%$; NHL, $21.8 \%$ ). The impact of reduced RDI on either disease-free or overall survival has been shown in both retrospective studies and prospective clinical trials. . $^{711,14,15}$

FN in the first cycle had a substantial impact on patient management, resulting in further dose reductions and delays along with increased use of CSF prophylaxis. Although the possible influence of the required midcycle blood draws on clinician behavior cannot be discounted, findings suggest that the dom- inant paradigm for managing neutropenic risk in the United States is to prescribe reduced chemotherapy dose intensity in the first cycle, with primary CSF prophylaxis also used in approximately a fifth of patients. In patients who received primary CSF prophylaxis, the incidence of neutropenic events was reduced in most cancers, consistent with the results of randomized clinical trials. ${ }^{20-23}$ Subsequently, more modest reductions in dose intensity and secondary CSF prophylaxis occured in later cycles from the occurrence of neutropenic events in the previous cycle.

The strengths and weaknesses of this study are important to consider. This study represents the largest prospective study of its kind, capturing consecutive unselected patients initiating a new chemotherapy regimen at 115 community oncology practices. Sites were randomly selected from all known community practices, after stratification, to obtain a representative sampling throughout the United States and participation from practices of all sizes. The nationwide distribution achieved closely matches the population density of the United States, and oncology practices across the full spectrum of size are included. Treatment regimens and supportive care represent the practice pattern at the included sites and are likely representative of the care received by cancer patients in community oncology practice..$^{24}$

Weaknesses of this trial include the likely underreporting of FN because only data for the first $3 \mathrm{cy}$ cles of treatment could be captured. The reported neutropenic event rates are probably underestimates, 


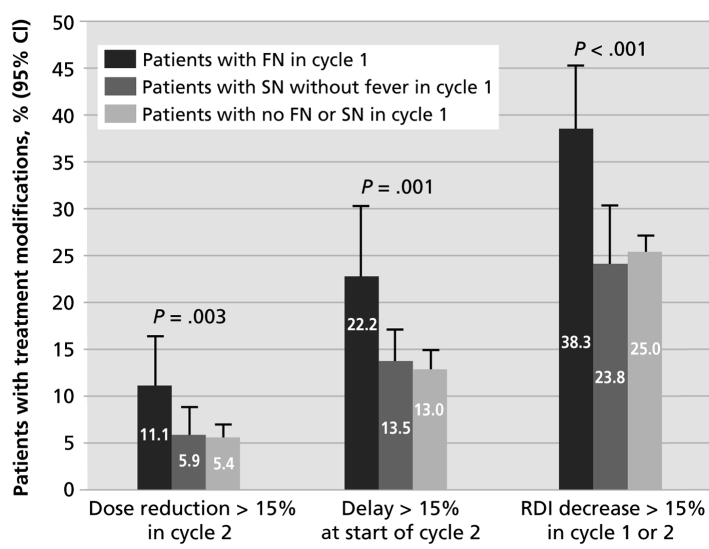

Figure 4 First-cycle FN (fever/infection and ANC $<1000 / \mathrm{mm}^{3}$ ) or SN (ANC $<500 / \mathrm{mm}^{3}$ without fever/infection) and incidence of dose reductions in cycle 2 , delays at the start of cycle 2 , and decreases in cycle 1 or $2 \mathrm{RDI}$. Dose reductions, treatment delays, and decreased RDI are defined as changes $>15 \%$ relative to the planned treatment. Abbreviations: ANC, absolute neutrophil count; CI, confidence interval; FN, febrile neutropenia; RDI, relative dose intensity; $\mathrm{SN}$, severe neutropenia.

because true nadirs may not have been captured for some patients, possibly resulting in lower rates of $\mathrm{SN}$ than actually occurred. In addition, a 19\% drop-out rate in patients between cycle 1 and 3 was seen, reflecting treatment discontinuation or patient loss from this observational study. Therefore, fewer neutropenic events may have been seen in later cycles both because higher-risk patients had been lost from the study and because treatment was changed to reduce neutropenic risk. Additional limitations of this study relate to the lack of specific information on the reasons for treatment and supportive care decisions and the lack of information on delayed toxicities and long-term outcomes. Finally, these data were collected before the current guidelines were available for CSF use from the NCCN, ${ }^{25}$ ASCO,${ }^{26}$ and EORTC, ${ }^{27}$ which may impact current and future clinical practice. However, given the variability in oncology practice shown in this registry, effective implementation of guidelines for CSF use will likely require more precise determination of patient-specific risks.

Assigning a specific risk to an individual patient remains empiric in practice, with the need for intervention based primarily on the perceived myelotoxicity of the prescribed regimen. ${ }^{28}$ The current guidelines for the use of myeloid growth factors uniformly call for improved assessment of neutropenic risk as an important means to increase both the quality of cancer care and the cost-effectiveness of preventive strategies. The registry data presented here clearly show that multiple patient-specific factors influence neutropenic risk, in concordance with those supported by retrospective evidence. ${ }^{16}$ A prospectively developed and validated predictive model based on these risk factors should enable providers to more precisely identify patients who are at increased risk and are therefore candidates for targeted intervention to reduce the risk. ${ }^{12,29}$ In addition to further reducing the incidence of FN, improved pretreatment assessment of neutropenic risk may help ensure that more patients are treated with optimal chemotherapy dose intensity, thereby giving them the best chance of achieving their treatment goals.

\section{Acknowledgment}

The authors would like to acknowledge and thank the study sites and investigators of the Awareness of Neutropenia in Chemotherapy (ANC) Study Group and patients included in this registry study for their participation.

\section{References}

1. Talcott JA, Siegel RD, Finberg R, Goldman L. Risk assessment in cancer patients with fever and neutropenia: a prospective, two-center validation of a prediction rule. J Clin Oncol 1992;148:2561-2568.

2. Kuderer NM, Dale DC, Crawford JC, et al. Mortality, morbidity, and cost associated with febrile neutropenia in adult cancer patients. Cancer 2006;106:2258-2266.

3. Hryniuk W, Bush $\mathrm{H}$. The importance of dose intensity in chemotherapy of metastatic breast cancer. J Clin Oncol 1984;2:1281-1288.

4. Link BK, Budd GT, Scott $S$, et al. Delivering adjuvant chemotherapy to women with early-stage breast carcinoma: current patterns of care. Cancer 2001;92:1354-1367.

5. Lyman GH, Dale DC, Crawford J. Incidence and predictors of low dose-intensity in adjuvant breast cancer chemotherapy: a nationwide study of community practices. J Clin Oncol 2003;21: 4524-4531.

6. Lyman GH, Dale DC, Friedberg J, et al. Incidence and predictors of low chemotherapy dose-intensity in aggressive non-Hodgkin's lymphoma: a nationwide study. J Clin Oncol 2004;22:4302-4311.

7. Kwak LW, Halpern J, Olshen RA, et al. Prognostic significance of actual dose intensity in diffuse large-cell lymphoma: results of a tree-structured survival analysis. J Clin Oncol 1990;8:963-977.

8. Epelbaum R, Faraggi D, Ben-Arie Y, et al. Survival of diffuse large cell lymphoma. A multivariate analysis including dose intensity variables. Cancer 1990;66:1124-1129.

9. Lepage $\mathrm{E}$, Gisselbrecht C, Haioun C, et al. Prognostic significance of received relative dose intensity in non-Hodgkin's lymphoma patients: application to LNH-87 protocol. The GELA (Groupe d'Etude des Lymphomes de l'Adulte). Ann Oncol 1993;4:651-656.

10. Bonadonna G, Valagussa $P$, Moliterni A, et al. Adjuvant cyclophosphamide, methotrexate, and fluorouracil in node-positive breast cancer: the results of 20 years follow-up. N Engl J Med 1995;332: 901-906. 
Crawford et al.

11. Budman DR, Berry DA, Cirrincione CT, et al. Dose and dose intensity as determinants of outcome in the adjuvant treatment of breast cancer. The Cancer and Leukemia Group B. J Natl Cancer Inst 1998;90:1205-1211.

12. Crawford J, Dale DC, Lyman GH. Chemotherapy-induced neutropenia: risks, consequences, and new directions for its management. Cancer 2004;100:228-237. Erratum in Cancer 2004;100:1993-1994.

13. Citron ML, Berry DA, Cirrincione $\mathrm{C}$, et al. Randomized trial of dosedense versus conventionally scheduled and sequential versus concurrent combination chemotherapy as postoperative adjuvant treatment of node-positive primary breast cancer: first report of Intergroup trial C9741/Cancer and Leukemia Group B trial 9741. J Clin Oncol 2003; 21:1431-1439.

14. Bonneterre J, Roche $\mathrm{H}$, Kerbrat $\mathrm{P}$, et al. Epirubicin increases longterm survival in adjuvant chemotherapy of patients with poor-prognosis, node-positive, early breast cancer: 10-year follow-up results of the French adjuvant study group 05 randomized trial. J Clin Oncol 2005;20:2686-2693.

15. Ardizzoni A, Favaretto A, Boni L, et al. Platinum-etoposide chemotherapy in elderly patients with small-cell lung cancer: results of a randomized multicenter phase II study assessing attenuated-dose or full-dose with lenograstim prophylaxis—a Forza Operativa Nazionale Italiana Carcinoma Polmonare and Gruppo Studio Tumori Polmonari Veneto (FONICAP-GSTPV) study. J Clin Oncol 2005; 23:569-575.

16. Lyman $\mathrm{GH}$, Lyman $\mathrm{CH}$, Agboola O. Risk models for predicting chemotherapy-induced neutropenia. Oncologist 2005;10:427-437.

17. Gomez H, Hidalgo M, Casanova L, et al. Risk factors for treatmentrelated death in elderly patients with aggressive non-Hodgkin's lymphoma: results of a multivariate analysis. J Clin Oncol 1998;16: 2065-2069.

18. Lyman GH, Morrison VA, Dale DC, et al. Risk of febrile neutropenia among patients with intermediate-grade non-Hodgkin's lymphoma receiving CHOP chemotherapy. Leuk Lymphoma 2003; 44:2069-2076.

19. Lyman GH, Delgado DJ. Risk and timing of hospitalization for febrile neutropenia in patients receiving $\mathrm{CHOP}, \mathrm{CHOP}-\mathrm{R}$, or $\mathrm{CNOP}$ chemotherapy for intermediate-grade non-Hodgkin lymphoma. Cancer 2003;98:2402-2409.

20. Vogel CL, Wojtukiewicz MZ, Carroll RR, et al. First and subsequent cycle use of pegfilgrastim prevents febrile neutropenia in patients with breast cancer: a multicenter, double-blind, placebo-controlled phase III study. J Clin Oncol 2005;23:1178-1184.

21. Crawford J, Ozer H, Stoller R, et al. Reduction by granulocyte colonystimulating factor of fever and neutropenia induced by chemotherapy in patients with small-cell lung cancer. N Engl J Med 1991;325: 164-170.

22. Trillet-Lenoir V, Green J, Manegold C, et al. Recombinant granulocyte colony stimulating factor reduces the infectious complications of cytotoxic chemotherapy. Eur J Cancer 1993;29A:319-324.

23. Timmer-Bonte JN, de Boo TM, Smit HJ, et al. Prevention of chemotherapy-induced febrile neutropenia by prophylactic antibiotics plus or minus granulocycyte colony-stimulating factor in smallcell lung cancer: a Dutch randomized phase III trial. J Clin Oncol 2005;23:7974-7984.

24. Warren JL, Harlan LC. Can cancer registry data be used to study cancer treatment? Medical Care 2003;41:1003-1005.

25. Crawford J, Althaus B, Armitage J, et al. Myeloid growth factors clinical practice guidelines in oncology. J Natl Compr Canc Netw 2005;3:540-555.

26. Smith TJ, Khatcheressian J, Lyman GH, et al. 2006 update of recommendations for the use of white blood cell growth factors: an evidence-based clinical practice guideline. J Clin Oncol 2006;24: 3187-3205.

27. Aapro MS, Cameron DA, Pettengell R, et al. EORTC guidelines for the use of granulocyte-colony stimulating factor to reduce the incidence of chemotherapy-induced febrile neutropenia in adult patients with lymphomas and solid tumours. Eur J Cancer 2006;42:2433-2453.

28. Dale DC, McCarter GC, Crawford J, et al. Myelotoxicity and dose intensity of chemotherapy: reporting practices from randomized clinical trials. J Natl Compr Canc Netw 2003;1:440-454.

29. Lyman GH. Guidelines of the National Comprehensive Cancer Network on the use of myeloid growth factors with cancer chemotherapy: a review of the evidence. J Natl Compr Canc Netw 2005;3:557-571. 